\title{
Endobronchial pulmonary blastoma - an unusual presentation of a rare lung malignancy and review of literature
}

\author{
Benhur Joel Shadrach¹, Deepak Vedant ${ }^{2}$, Vikarn Vishwajeet ${ }^{2}$, Priyank Jain ${ }^{1}$, Naveen Dutt ${ }^{1}$, Binit Surekha ${ }^{3}$, \\ Puneet Pareek ${ }^{4}$, Poonam Abhay Elhence ${ }^{2}$ \\ ${ }^{1}$ Department of Pulmonary Medicine; ${ }^{2}$ Department of Pathology; ${ }^{3}$ Department of Radiodiagnosis; ${ }^{4}$ Department of \\ Radiation Oncology, All India Institute of Medical Sciences, Jodhpur, India
}

\begin{abstract}
Biphasic pulmonary blastoma (BPB) is an extremely rare highly aggressive malignant tumour that arises from fetal lung tissue and has the classical biphasic histology of epithelial and mesenchymal components. It is usually seen in adults with a slight
\end{abstract}

Correspondence: Deepak Vedant, Department of Pathology, All India Institute of Medical Sciences, Jodhpur, India.

Tel. +9882424889

E-mail: dr.tomchi@gmail.com.

Key words: Pulmonary blastoma; endobronchial; histology; prognosis.

Contributions: BJS, DV, VV, PJ, ND, BS, PP, PAE, concepts; BJS, DV, VV, PJ, ND, BS, design; BJS, DV, PJ, PP, PAE, definition of intellectual content; BJS, DV, PJ, ND, PP, literature research; BJS, VV, PJ, ND, manuscript preparation; BJS, DV, PJ, BS, PP, PAE, manuscript editing; BJS, DV, VV, BS, PP, PAE, manuscript review; BJS, $\mathrm{BS}, \mathrm{PAE}$, guarantors.

Conflict on interest: The authors declare no conflict of interest.

Funding: None.

Availability of data and materials: All data underlying the findings are fully available.

Ethics approval and consent to participate: No ethical committee approval was required for this case report by the Department, because this article does not contain any studies with human participants or animals. Informed consent was obtained from the patient included in this study.

Consent for publication: The patient gave his written consent to use his personal data for the publication of this case report and any accompanying images.

Received for publication: 18 June 2020.

Accepted for publication: 19 June 2020.

${ }^{\circ}$ Copyright: the Author(s), 2020

Licensee PAGEPress, Italy

Monaldi Archives for Chest Disease 2020; 90:1462

doi: 10.4081/monaldi.2020.1462

This article is distributed under the terms of the Creative Commons Attribution Noncommercial License (by-nc 4.0) which permits any noncommercial use, distribution, and reproduction in any medium, provided the original author(s) and source are credited. predominance among males and smokers. Previously grouped along with well-differentiated fetal adenocarcinoma (WDFA), and pleuropulmonary blastoma (PPB), now it is considered a separate variant and grouped under sarcomatoid neoplasms. Symptoms include chest pain, cough, hemoptysis and it is asymptomatic in at least one-third of the cases. A biopsy is essential for diagnosis and surgical excision is the treatment of choice. Prognosis is poor with 5-year survival less than $20 \%$ and recurrence occurring within 12 months of surgery. An aggressive multimodality approach is required for its management and active follow up surveillance is needed to look for recurrence.

\section{Introduction}

Biphasic pulmonary blastoma (BPB) is a rare lung neoplasm comprising $0.25-0.5 \%$ of primary lung malignancies with a very aggressive course and poorer prognosis $[1,2]$. It was initially described by Barnett et al. [3] in 1952 and since then approximately 300 cases have been reported in the literature [4]. Morphologically, it resembles fetal lung tissue of gestation age less than 16 weeks and has typical biphasic histology (epithelial and mesenchymal components) [5]. Despite having fetal lung tissue origin, it is seen predominantly in adults [2]. Due to its rarity, non-specific clinical symptoms, subtle radiological findings, variable histology, rapid progression, and poor prognosis, the diagnosis and management is challenging. Here, we describe a rare case of a classical BPB with endobronchial spread and a brief review of the literature.

\section{Case Report}

A 30-year-old Indo-Aryan male, chronic smoker, with no previous co-morbidities was admitted to our hospital with complaints of cough with minimal expectoration, right-sided chest pain, and shortness of breath on exertion for the last four months. He also had multiple episodes of mild hemoptysis in the last two months. On admission, he had tachycardia, tachypnoea with accessory respiratory muscle use, was normotensive and had slightly reduced peripheral oxygen saturation $\left(\mathrm{SpO}_{2}-93 \%\right.$ on room air). Arterial blood gas (ABG) on ambient air revealed respiratory alkalosis and mild hypoxemia $\left(\mathrm{PaO}_{2}-70 \mathrm{~mm} \mathrm{Hg}\right)$. Respiratory system examination disclosed decreased chest expansion and absent breath sounds in the right hemithorax. Chest radiograph showed an opaque right hemithorax (Figure 1A). Contrast-enhanced computerized tomog- 
raphy (CECT) of the thorax revealed a right-sided large homogeneous mass $(9.3 \mathrm{~cm})$ with endobronchial spread involving right main bronchus causing an ipsilateral mediastinal shift and mediastinal lymphadenopathy (Figure 1B). After obtaining informed consent, ultrasound-guided (USG) transthoracic biopsy was done. However, transthoracic biopsy was non-contributory.

Video bronchoscopy and endobronchial biopsy was performed. White light bronchoscopy (WLB) revealed a fleshy endobronchial mass in the right main bronchus (RMB) (Figure $2 \mathrm{~A}, \mathrm{~B}$ ). Multiple endobronchial biopsies were taken and sent for histopathology (HPE). Hematoxylin and Eosin (H\&E) stained sections showed a tumour with biphasic morphology comprised of irregular elongated glands and cellular mesenchymal component. The glands are lined by low to tall columnar, focally stratified cells with hyperchromatic nuclei, inconspicuous nucleoli and scanty cytoplasm. Few small supra-nuclear vacuoles are noted. The mesenchymal component shows nuclear pleomorphism with irregular hyperchromatic nuclei and scanty cytoplasm (Figure 3 A,B). Focal stromal vacuolisation is noted. On immunohistochemistry (IHC), the malignant glandular component displays thyroid transcription factor-1 (TTF-1) nuclear immuno-positivity (Figure 3C) indicating pulmonary origin and is negative for alpha-smooth muscle actin (SMA) and vimentin while the malignant mesenchymal component is immuno-positive for vimentin (Figure 3D) and alpha-SMA (Figure 3E) and negative for TTF-1. Hence, a diagnosis of pulmonary blastoma was rendered.
Whole-body positron emission tomography-computed tomography (PET-CT) was done for staging and resectability status. PET-CT showed a dense FDG avid large homogeneous mass in the right hemithorax measuring $10.8 \mathrm{~cm}$ with SUV uptake 14.8 $\mathrm{HU}$ and FDG avid right paratracheal lymph node (SUV- 11.1HU) (Figure 4 A,B). Staging was done similar to non-small cell cancer (NSCLC) and was labelled as stage III-B (T4N2M0) [6]. An integrated multimodality approach involving a team of Pulmonologists, Oncophysicians, and Oncosurgeons was formed and a multidisciplinary discussion (MDD) was initiated to decide on further management. In view of TNM stage III-B and involvement of carina (a relative contraindication for pneumonectomy), it was decided to initiate neoadjuvant chemotherapy and reassess for surgery after 3 cycles of chemotherapy. The patient and his family were explained regarding the decision of the MDD. He was put on conventional chemotherapy regimen (cisplatin and etoposide) and asked to follow up for after completing 3 cycles of chemotherapy.

\section{Discussion}

Pulmonary blastomas are unusual primary lung malignancies comprising less than $1 \%$ of all lung tumours. It was poorly understood because of its biological behaviour and initially referred to as

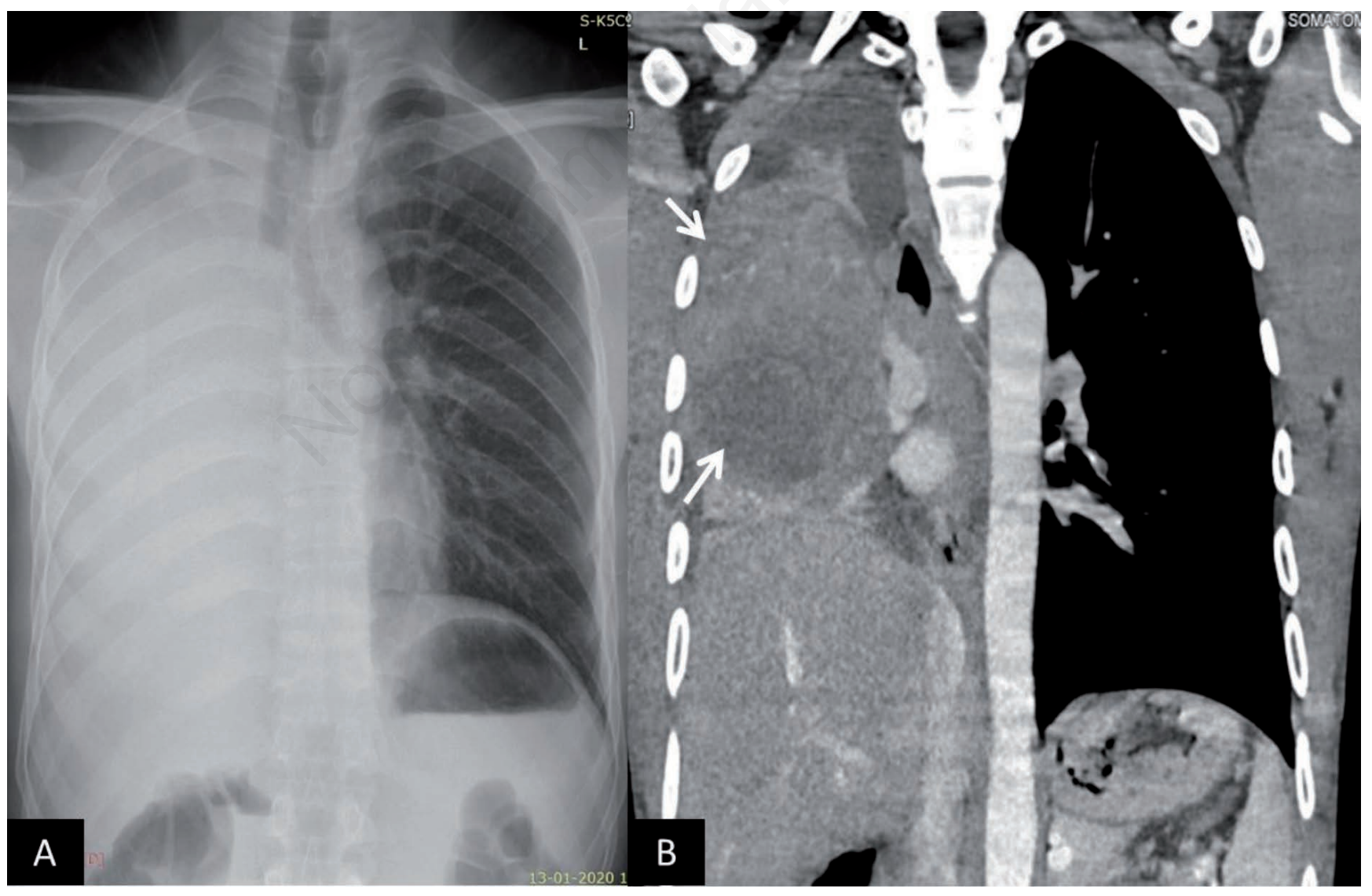

Figure 1. A) Chest radiograph showing opaque right hemithorax with mediastinal shift. B) Coronal reformatted CT images showing heterogeneously enhancing large mass lesion occupying almost the right hemithorax (arrows) with infiltration into the right main bronchus and causing passive collapse of underlying right lung. 


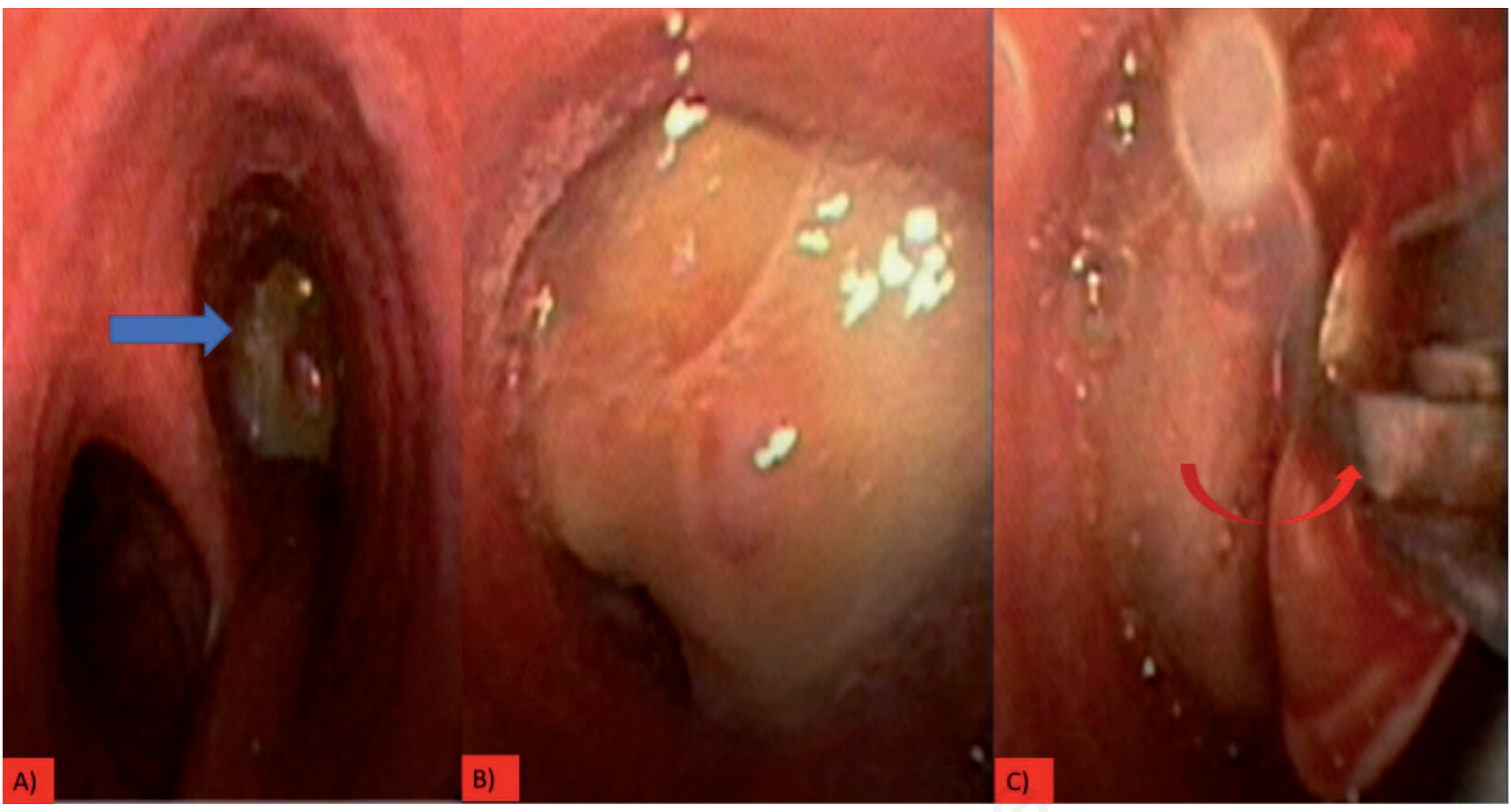

Figure 2. A,B) White light bronchoscopy revealing a fleshy endobronchial mass in right main bronchus (blue arrow). C) Endobronchial biopsies taken with fenestrated cup forceps (red arrow).

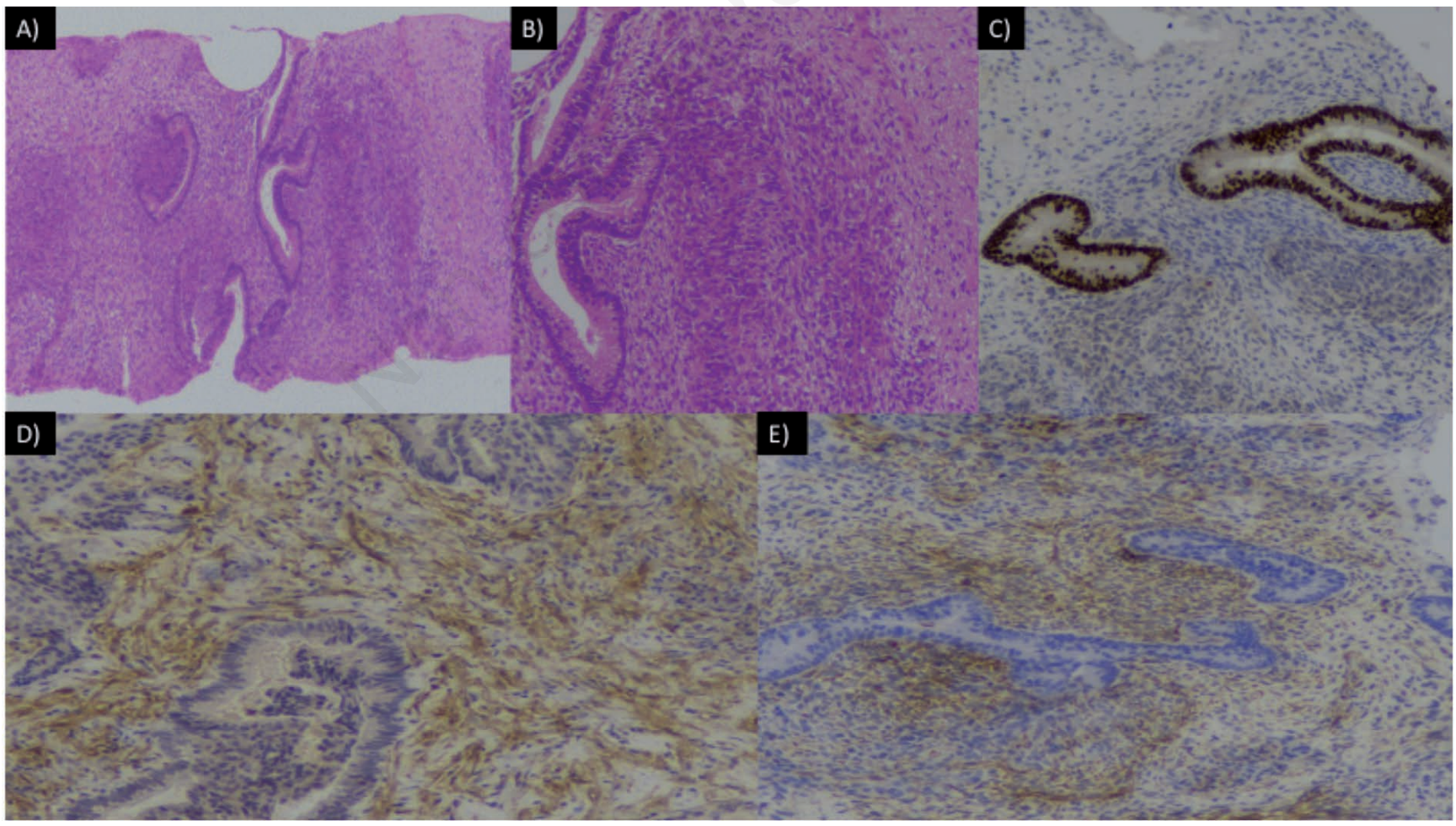

Figure 3. A) Biopsy showing an admixture of elongated irregular glands and cellular mesenchymal component; the glands are lined by columnar cells with tall columnar hyperchromatic nuclei, H\&E stain, 4x. B) Biopsy showing glands lined by hyperchromatic focally stratified nuclei with few supranuclear vacuolations, H\&E stain, 10x. C) IHC for TTF1 showing intense nuclear immunopositivity, DAB-H, 10x. D) IHC for vimentin showing cytoplasmic positivity in the mesenchymal component, DAB-H, 10x. E) IHC for alpha-SMA showing cytoplasmic positivity in the mesenchymal component, DAB-H, 10x. 


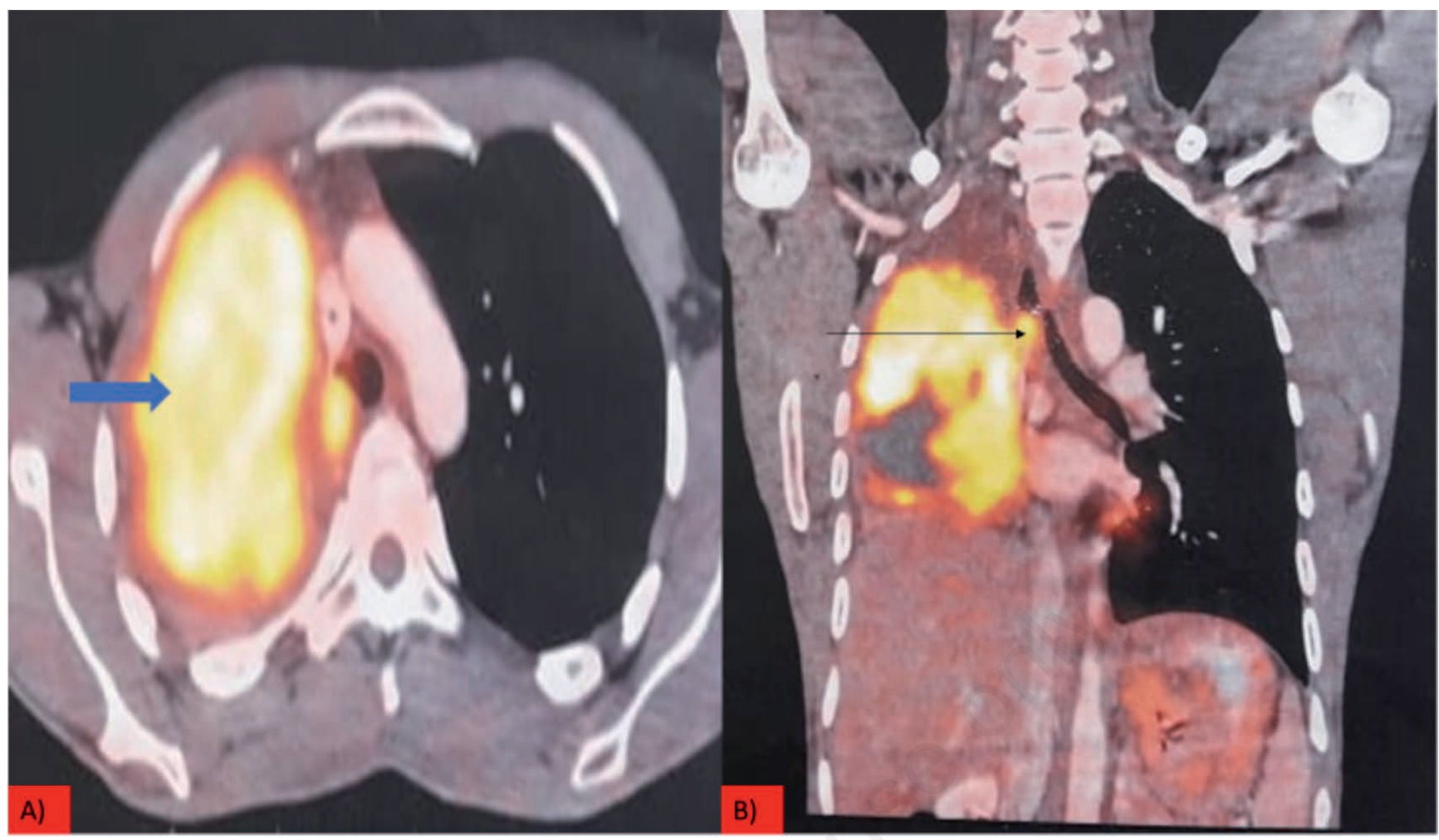

Figure 4. A) PET-CT showing FDG avid mass with ipsilateral paratracheal adenopathy (blue arrow). B) Coronal reformatted PET-CT image confirming right bronchial tree and carinal involvement (black arrow).

an embryoma [3]. Later it was found that the tumour arises from fetal lung tissue and was labelled as pulmonary blastemas. This was further classified into 3 subtypes by Koss et al. [7]. These include biphasic pulmonary blastoma, monophasic pulmonary blastoma with predominant epithelial expression (WDFA), and pleuropulmonary blastoma (PPB) with mesenchymal expression. WHO classifications (1999 and 2004) separated BPB from WDFA and PPB and are now grouped under sarcomatoid carcinomas [8]. Classical BPB typically occurs in adults with an average age of onset at 40 years with a slight male predisposition (2:1) [9]. There are no identifiable risk factors, but there appears to be a strong correlation with cigarette smoking [10]. Nearly $40 \%$ of cases are asymptomatic and detected during incidental imaging [7]. Among symptomatic cases, common symptoms include cough, hemoptysis, chest pain and dyspnea due to tumour compressing the bronchi and/or pleura. Although pleural and chest wall extension have been described in the literature [11], endobronchial involvement has not been reported previously to the best of our knowledge.

Radiology shows a large well defined homogenous solitary mass with smooth margins, almost always unilateral and peripherally located close to the pleura [12]. Due to its proximity with the pleura, bronchoscopy and biopsy is diagnostic in only onefourth of the cases. Ultrasound/CT guided transthoracic biopsy serves as an excellent modality in the diagnosis [13]. Histopathological diagnosis of BPB is difficult due to its pleomorphic histology but requires a high degree of suspicion when there is cytological heterogeneity with a mixture of malignant epithelial and mesenchymal cells [5]. There is no separate staging system for pulmonary blastoma and it is reasonable to stage them in the same way as NSCLC. Distant metastasis by hematogenous spread to brain, bone, liver, kidney, pancreas, and adrenal glands has been described in the literature. The role of PET-CT is not well studied and may help predict operability and relapse after surgical excision. Pulmonary blastomas are intensely FDG-avid which could be attributed to the presence of fetal lung tissue [14].

Surgery is the treatment of choice and complete surgical excision is the only curative treatment [15]. A mean survival of 33 months was noted after surgical excision compared to less than 2 months in unresected disease. In surgery, Larsen and colleagues [9] found that local resection had a better survival than pneumonectomy. Blastomas are relatively chemo- and radio-resistant and may be useful in the relief of palliative symptoms [2]. Chemotherapy had a mere $16 \%$ response rate [9]. However, neoadjuvant chemotherapy combined with postoperative radiotherapy and chemotherapy have shown a better prognosis [16] Among chemotherapeutic agents, cisplatin and etoposide are preferred. Recently, a combination of ifosfamide, carboplatin, and etoposide popularly known as the ICE protocol was used as an effective treatment of pulmonary blastoma. Complete remission was noted at 3 months follow up after 6 cycles of ICE [17]. BPB has a poor prognosis with nearly $2 / 3$ of patients die within 2 years and an estimated 5-year survival is $15-30 \%$ [2,10]. Factors responsible for poor prognosis include tumour size $>5 \mathrm{~cm}$, metastasis, completeness of resection and tumour recurrence. The recurrence rate is high the first year after resection with nearly $43 \%$ of cases have a recurrence and after one year, recurrence usually does not occur [18]. 


\section{Conclusions}

Our report highlights an atypical presentation of adult pulmonary blastoma and alerts pulmonologists the importance of an integrated multimodality approach. Surgical excision is curative and regular follow up is advised. In inoperable cases, conventional chemotherapy or the ICE regimen can be considered. Despite the best management, prognosis is poor and inversely related with the delay in diagnosis.

\section{References}

1. Bisogno G, Brennan B, Orbach D, et al. Treatment and prognostic factors in pleuropulmonary blastoma: an expert report. Eur J Cancer 2014;50:178-4.

2. Want Y-X, Zhang J, Chu X-Y, et al. Diagnosis and multimodality treatment of adult pulmonary blastoma: Analysis of 18 cases and review of the literature. Asian Pac J Trop Med 2014; 7:164-8.

3. Barnett N, Barnard W. Some unusual thoracic tumors. Br J Surg 1945;32:441-57.

4. Jethava A, Dasanu C. Adult biphasic pulmonary blastoma. Conn Med 2013;71:19-22.

5. Magistrelli P, D'Ambra L, Berti S, et al. Adult pulmonary blastoma: report of an unusual malignant lung tumor. World J Clin Oncol 2014;5:1113-6.

6. Goldstraw P, Chansky K, Crowley J, et al. The IASLC lung cancer staging project: proposals for revision of the TNM stage groupings in the forthcoming (eighth) edition of the TNM classification for lung cancer. J Thorac Oncol 2016;11:39-51.
7. Koss MN, Hochholzer L, O'Leary T. Pulmonary blastomas. Cancer 1991;67:2368-81.

8. Daghfous H, Belloumi N, Braham E, et al. Case report of pulmonary biphasic tumor with unusual follow-up. Rev Pneumol Clin 2014;70:302-6.

9. Smyth RJ, Fabre A, Dodd JD, et al. Pulmonary blastoma: a case report and review of the literature. BMC Res Notes 2014;7:294.

10. Larsen H, Sorensen JB: Pulmonary blastoma: a review with special emphasis on prognosis and treatment. Cancer Treat Rev 1996;22:145-60.

11. Liman ST, Altinok T, Topcu S, et al. Survival of biphasic pulmonary blastoma. Respir Med 2006;100:1174-9.

12. Dixit R, Joshi N, Dave L. Biphasic pulmonary blastoma: An unusual presentation with chest wall, rib, and pleural involvement. Lung India 2014;31:87-9.

13. Robert J, Pache J-C, Seium Y, et al. Pulmonary blastoma: report of five cases and identification of clinical features suggestive of the disease. Eur J Cardiothorac Surg 2002;22:708-1.

14. Bini A, Ansaloni L, Grani G, et al. Pulmonary blastoma: report of 2 cases. Surg Today 2001;31:438-2.

15. Keu KV, Berry GJ, Quon A. Classic biphasic pulmonary blastoma demonstrated by $18 \mathrm{~F}-\mathrm{FDG}$ PET/CT. Clin Nucl Med 2014;39:346-8

16. Mistry JH, Pawar SB, Mehta H, et al. Primary pulmonary blastoma of monophasic variety--diagnosis and management. J Cardiothorac Surg 2013;8:144.

17. Iwata $T$, Nishiyama $N$, Inoue $K$, et al. Biphasic pulmonary blastoma: report of a case. Ann Thorac Cardiovasc Surg 2007; $13: 40-3$.

18. Alahwal MS, Maniyar IH, Saleem F, Alshiekh M. Pulmonary blastoma: a rare primary lung malignancy. Case Rep Med $2012 ; 471613$. 